\title{
Coarctation of the aorta in prenatal life: an echocardiographic, anatomical, and functional study
}

\author{
LINDSEY D ALLAN, * SUNDER K CHITA, * ROBERT H ANDERSON, $\dagger$ \\ NUALA FAGG, $\ddagger$ DIANE C CRAWFORD, * MICHAEL J TYNAN * \\ From the ${ }^{\star}$ Departments of Paediatric Cardiology and $\ddagger$ Pathology, Guy’s Hospital; and the $†$ Cardiothoracic \\ Institute, Brompton Hospital, London
}

SUMMARY In a prospective echocardiographic study of over 2000 pregnancies, 24 fetuses were found to have dilatation of the right ventricle and pulmonary artery when these structures were compared with those of the left heart. In 18 of these cases the diagnosis of coarctation or interruption of the aorta was correctly inferred from these findings. On direct echocardiographic examination of the aortic arch, arch hypoplasia or interruption of the aortic arch was recognisable prenatally in 10 of these 18 cases, most readily between 20-30 weeks' gestation. In 11 of 12 cases of aortic arch anomaly studied by Doppler echocardiography, a reduction in blood flow in the ascending aorta was demonstrated. The intracardiac appearances were suggestive of coarctation but the Doppler findings were within normal limits in two further cases which later proved to be normal. A diaphragmatic hernia was found in four fetuses and the intracardiac echocardiography and Doppler findings were the same as those found in fetuses with aortic coarctation. Five cases of coarctation were overlooked on the prenatal echocardiogram but these were found at follow up of the infants.

Thus coarctation of the aorta can be diagnosed prenatally, although the condition may be missed or incorrectly predicted by the criteria examined in the present study. Reduced aortic blood flow is a demonstrable feature of aortic arch abnormalities but it is found in other conditions that are not associated with coarctation.

During our total experience of fetal echocardiography we have had the opportunity to describe both normal and abnormal cardiac anatomy ${ }^{12}$ and to follow the development of cardiac structure in the normal fetus. ${ }^{3}$ More recently we have been able to define the normal flow patterns during fetal life. ${ }^{4}$ Earlier in this programme we reported on the echocardiographic diagnosis and evolution of a case of coarctation of the aorta recognised initially at 20 weeks' gestation. ${ }^{5}$ This case showed that coarctation of the aorta could be diagnosed prenatally but raised questions about the prevailing theories of its development. This case prompted a prospective study of the diagnosis of fetal coarctation in 2000 pregnancies that we subsequently examined and in which we studied development of the heart and great arteries in those suspected cases which went to term.

Requests for reprints to Dr Lindsey D Allen, Department of Perinatal Cardiology, 15th Floor, Guy's Tower, Guy's Hospital, St Thomas's Street, London SE1 9RT.

Accepted for publication 19 November 1987

\section{Patients and methods}

We used either an Advanced Technical Laboratories Mark III or a Hewlett-Packard 77020A sector scanner to study 2029 fetuses. The fetal echocardiography programme including Doppler investigation was approved by the ethics committee and each mother gave informed consent to the study. The whole echocardiogram was stored on videotape. The examination was repeated if all the necessary infor- $\tilde{\sigma}$ mation could not be obtained at one sitting or if an abnormality was found and the pregnancy continued.

Neonates were examined by a paediatric cardiologist or paediatrician. All fetuses that died in utero and the products of termination were examined at necropsy.

The echocardiographic signs that we particularly noted were: $(a)$ relative sizes of the right and left ventricles as measured at the level of the atrioventricular valve orifices in a frozen frame diastolic image of a four chamber view of the fetal heart (the range (mean (1 SD)) for this ratio, based on 80 ? normal fetuses is $\left.1.1(0.09)^{4}\right)$; $(b)$ relative sizes of the 8 
pulmonary artery and aorta. These measurements were made at the origin of each vessel with the image frozen in systole. The range (mean (1 SD)) for this ratio based on 80 normal fetuses is $1 \cdot 2(0 \cdot 18) ;(c)$ the appearance of the aortic arch and isthmus. This was a subjective evaluation of the arch structures in relation to the sizes of adjacent vessels such as the arterial duct and branch pulmonary arteries.

When any intracardiac or aortic arch abnormality was suspected we also examined the atrioventricular and arterial valves by Doppler echocardiography when this facility became available. Blood flow was then estimated as previously described. ${ }^{4}$

\section{Results}

Twenty four of the 2029 fetuses had relative right ventricular and pulmonary artery dilatation as judged by cross sectional echocardiography. The diagnosis of coarctation was suspected from these signs in 16 cases. In no case could a discrete coarctation "shelf" itself be positively identified on direct echocardiographic examination of the arch, but convincing arch hypoplasia could be identified in eight patients. A discrete shelf lesion was, however, found at postnatal operation in two, at necropsy in 10 , and at both in four. In two further cases, the intracardiac findings were the same as those with coarctation but the arch was seen to be interrupted. A prospective echocardiographic diagnosis of aortic arch abnormality was correctly made, therefore, in 18 of the 24 cases.

In 17 of the 24 patients studied by Doppler three had two studies and one had three studies. Tricuspid flow was more than twice mitral flow in all 22 examinations. Blood flow in the ascending aorta was measured in 20 studies. This measurement was below the normal range for the gestation in 12 and

Table Prospective diagnoses

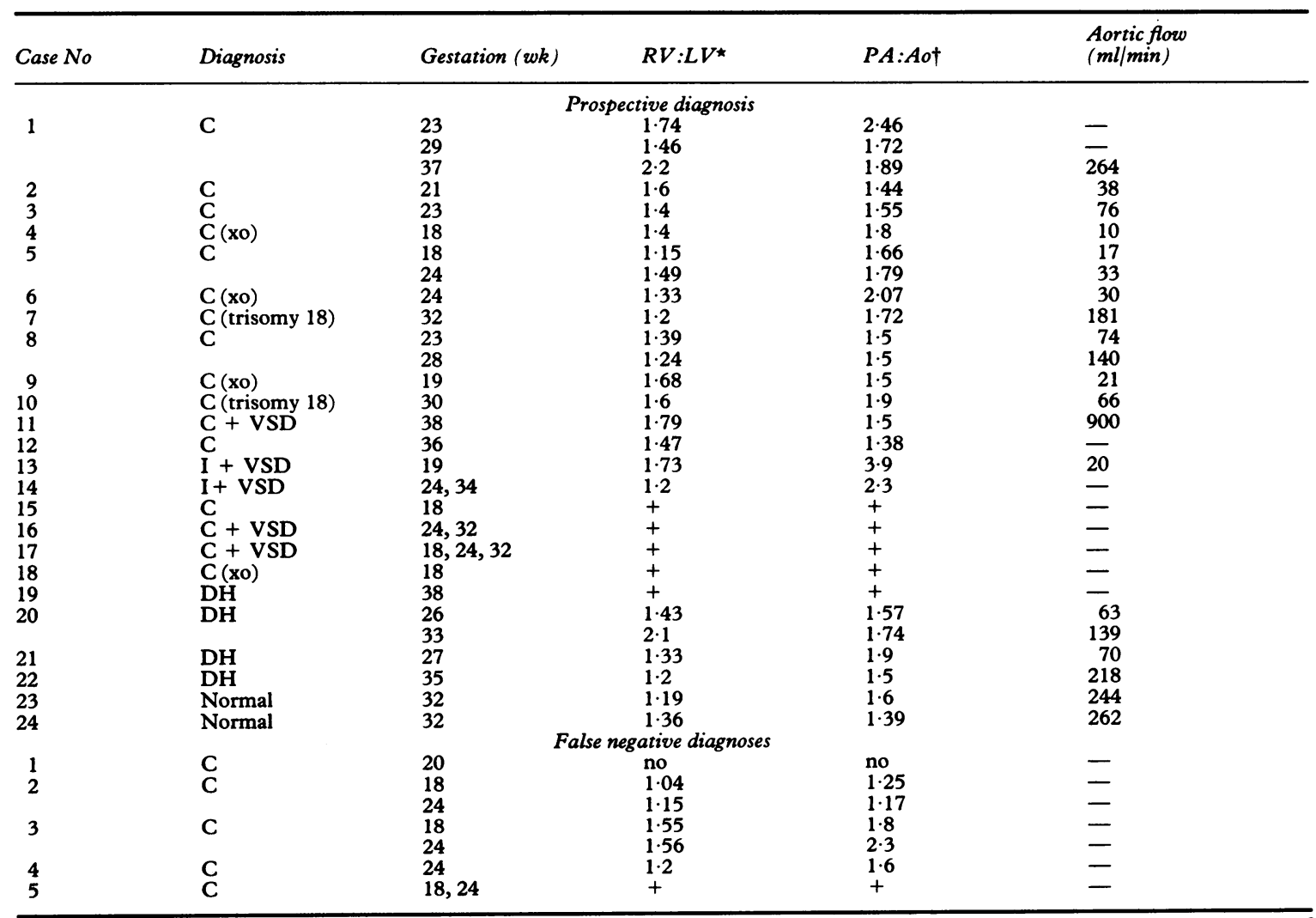

+ The videotape was recorded on equipment from which retrospective measurements cannot be made but on gross examination an increase of the RV:LV and PA:Ao ratios was apparent.

^Normal range $1 \cdot 1(0.09)$.

†Normal range $1 \cdot 2(0 \cdot 18)$.
C, coarctation; $I$, interruption; VSD, ventricular septal defect; xo, Turner's syndrome. 


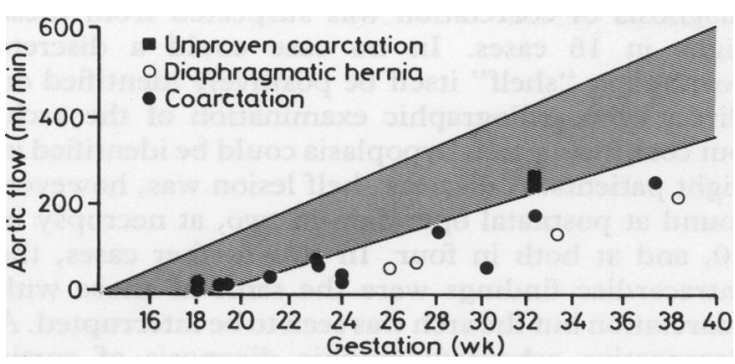

Fig 1 Relation between aortic blood flow and gestational age. The shaded area shows the normal range. The values for each of the abnormal groups are shown. In both real aortic arch lesions and in diaphragmatic hernias the aortic flow is significantly below the normal mean ( $p>0.001)$.

below the mean in a further five examinations. Doppler examination showed reduced blood flow in the aorta in 11 of 12 fetuses with confirmed coarctation or interruption of the aorta. Figure 1 shows aortic flow in each of these studies. In one fetus with coarctation, the aortic flow was much greater than normal mean value (case 11 in table).

In four of the 24 cases the fetal scan showed a diaphragmatic hernia (fig 2). Three of these had both anatomical and flow features consistent with coarctation. In one only the anatomical features were known because Doppler was not available at the time of examination. Underdevelopment of the left heart is known to occur with diaphragmatic hernia. ${ }^{67}$ These intracardiac findings were therefore considered to be secondary to the diaphragmatic lesion.

Two fetuses had cross sectional echocardiographic appearances suggesting coarctation but without any abnormality of the aortic arch on direct examination. It was felt that in these two cases a diagnosis of coarctation could not be excluded. The Doppler flow patterns were within normal limits in both cases. These two fetuses were normal at postnatal follow up.

The table shows ages at which the echocardiographic abnormalities were detected. The intracardiac signs were detected at presentation irrespective of gestational age. The aortic arch was difficult to assess conclusively in the youngest and the oldest fetuses. Of the total group of 18 cases of arch abnormality, eight aortic arches were identified as hypoplastic and two were seen to be interrupted. In a further three cases, the arch was not seen adequately for assessment. In the remaining five cases the appearance of the arch was within normal limits. In the seven cases of coarctation studied serially to term the size of the arch and isthmus relative to other cardiac structures decreased towards term in three. The earliest that coarctation or interruption was suspected on cross sectional echocardiography was 18 weeks, and seven of the abnormal fetuses were detected by 20 weeks. The abnormal aortic flow pattern, however, was more convincingly outside the normal range in studies after 20 weeks' gestation (fig 1).

Coarctation was not diagnosed prospectively in five of the 2029 patients. These cases presented with this condition after birth. Review of the fetal echocardiogram showed normal cross sectional echocardiographic features in two. The features of coarctation had been overlooked in the other three. Doppler interrogation of all the cardiac valves had not been performed in these five cases because in our centre this is not a routine investigation unless coarctation is suspected antenatally.

\section{Discussion}

Our results show that it is possible to recognise coarctation of the aorta as early as 18 weeks' gestation. Recognition depends on integrating many features. These include analysis of the relative sizes of the two ventricles and great arteries and the evaluation of the aortic arch in fetal life. The right ventricle is normally slightly larger than the left and the pulmonary artery slightly larger than the aorta. ${ }^{4}$ If these features are more pronounced than normal coarctation should be suspected. In the past the evaluation of the arch and isthmus relative to the arterial duct and pulmonary arteries was subjective. Recent measurements of these structures in the normal fetus will allow us to be more objective in

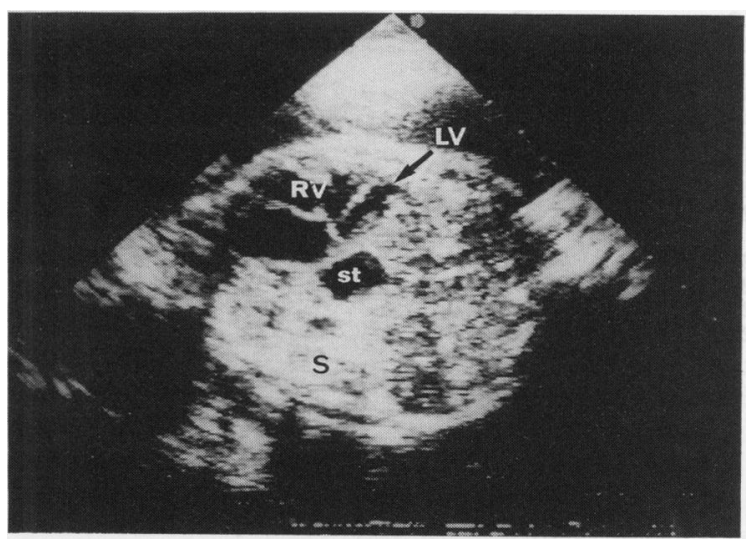

Fig 2 The heart in the four chamber projection showing the relative hypoplasia of the left ventricle ( $L V)$ that is caused by the stomach and bowel occupying the whole of the left thorax. The heart lies in the right chest. s, spine; st, stomach; $R V$, right ventricle. 


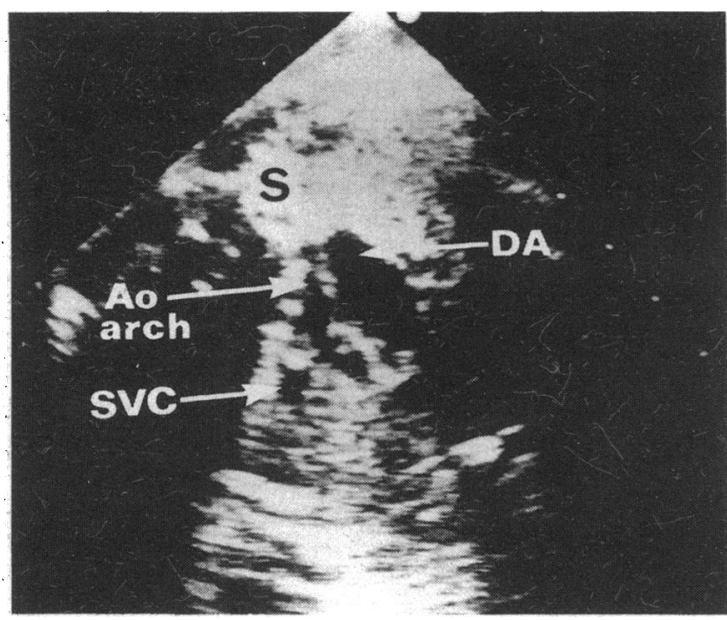

Fig 3 The diameter of the crest of the aortic (Ao) arch in this patient is much smaller than that of ductus arteriosus (DA). There was severe arch hypoplasia in association with coarctation in this case. SVC, superior vena cava.

future (unpublished observations). Measurement of arch structures may also help to identify those cases with less severe arch hypoplasia because some hypoplasia is normal. If diminished aortic flow is recorded on Doppler the likelihood of coarctation is increased. The combined findings give a high degree of accuracy.

On occasion, the relative sizes of the ventricles and great arteries in late pregnancy may suggest coarctation and it may be difficult to visualise the aortic arch. Previously, we sought the longitudinal view of the fetal arch for examination. This is similar to the view seen postnatally. This was difficult to obtain in every patient, especially in late pregnancy or in some cases where the fetus was lying in an infavourable position. Latterly, we have analysed horizontal views of the thorax, which allow us to see the arch and arterial duct in the same section. ${ }^{8}$ This permits direct comparison of the size of these structures. The crest of the aortic arch and the arterial duct should be of similar size. Figure 3 shows one of the cases of coarctation where the arch was severely hypoplastic. This view of the arch is more readily obtainable in all fetuses, irrespective of fetal age or position. Use of this approach may improve our record of adequate examination of this site.

There was $>2: 1$ flow across the tricuspid and mitral valves in all cases of coarctation or interruption of the aorta. The upper limit of the ratio in the normal fetus is $1 \cdot 8: 1$. $^{4}$ In the two cases of suspected coarctation absolute aortic flow measurement established that these were in fact normal cases.
In the five false negative diagnoses of coarctation, retrospective examination of the videotaped echocardiogram showed normal anatomical features in two cases (cases 1 and 2 in table). One of these cases presented without symptoms after the neonatal period, while the other had a discrete coarctation with no isthmal narrowing. This raises the possibility of two forms of coarctation, one being haemodynamically important in fetal life and usually associated with isthmal hypoplasia, and the other arising in postnatal life, perhaps produced by or dependent on ductal closure for its complete development. All the cases of coarctation predicted prenatally had a degree of isthmal hypoplasia postnatally or at necropsy whether this feature had been clearly recognised echocardiographically or not. Those cases with severe arch hypoplasia were most readily recognisable on the prenatal echocardiogram.

Echocardiographic and Doppler findings in four cases of diaphragmatic hernia were similar to the findings in coarctation. We attributed the low flow through the left heart with the consequent right ventricular and pulmonary artery dilatation to left heart compression by the diaphragmatic hernia. An alternative explanation is that the diminished pulmonary venous return caused by lung hypoplasia may reduce flow through the left ventricle and thus lead to underdevelopment of this chamber. The fact that in these cases a major disturbance of left ventricular flow with appreciable decrease in the size of the arch was not associated with coarctation casts doubt upon the flow dependent causation of coarctation. One of the cases of coarctation had aortic flow well above the normal range. This fetus had a large ventricular septal defect and severe right ventricular dysfunction, which may account for this finding.

We conclude that coarctation of the aorta can be diagnosed by prenatal echocardiography. This finding challenges the proposed role of the closure of the arterial duct in the development of aortic coarctation. False positive diagnosis, and perhaps also false negative diagnosis, can be avoided if Doppler evaluation of aortic blood flow is included in the fetal examination. It may not be possible, however, to recognise prenatally some milder forms of coarctation. The degree of decrease in aortic blood flow may contribute to the severity of arch hypoplasia occurring in association with coarctation. The reduced aortic flow none the less, must be secondary to the presence of the anatomical shelf lesion because other conditions occurring in utero that produce diminished aortic blood flow, such as diaphragmatic hernia, do not lead to development of coarctation.

The work was supported by the British Heart Foundation and the Joseph Levy Foundation. 


\section{References}

1 Allan LD, Tynan MJ, Campbell S, Wilkinson JL, Anderson RH. Echocardiographic and anatomical correlates in the fetus. Br Heart $J$ 1980;44:444-51.

2 Allan LD, Crawford DC, Anderson RH, Tynan MJ. Echocardiographic and anatomical correlations in fetal congenital heart disease. $\mathrm{Br}$ Heart $J$ 1984; 52:542-8.

3 Allan LD, Joseph MC, Boyd EGCA, Campbell S, Tynan M. M-mode echocardiography in the developing human fetus. Br Heart $J$ 1982;47:573-83.

4 Allan LD, Chita SK, Al-Ghazali W, Crawford DC, Tynan M. Doppler echocardiographic evaluation of the normal human fetal heart. Br Heart 1987;57:528-33.

5 Allan LD, Crawford DC, Tynan M. Evolution o $\overrightarrow{\vec{B}}$ coarctation of the aorta in intrauterine life. Br Heart $\delta$ 1984;52:471-3.

6 Siebert JR, Hass JE, Beckwith JB. Left ventriculare hypoplasia in congenital diaphragmatic hernia $\widehat{\Phi}$ $J$ Pediatr Surg 1984;19:567-71.

7 Crawford DC, Drake DP, Kwaitkowski D, Chapmanis MG. Allan LD. Prenatal diagnosis of reversible cardiac hypoplasia associated - with congenitaL diaphragmatic hernia: Implications for postnata $\vec{\omega}$ management. JCU 1986;14:718-21.

8 Allan LD. Manual of fetal echocardiography. Lancaster: MTP Press, 1986:18-44. 